\title{
Comparison of two sialosyl-Tn binding monoclonal antibodies (MLS102 and B72.3) in detecting pancreatic cancer
}

\author{
C K Ching, S W Holmes, G K T Holmes, R G Long
}

\begin{abstract}
An immunohistochemical study has been carried out to compare and contrast the cellular distribution of two different sialosylTn antigen binding monoclonal antibodies, MLS102 and B72.3, in the pancreas. MLS102 but not B72.3 monoclonal antibody binding increases with the content of the sialosyl-Tn epitopes. It was found that all 13 pancreatic cancer specimens bound both MLS102 and B72.3 monoclonal antibodies. Their cellular distribution in the cancer was virtually identical. Fifteen of $20(75 \%)$ patients with chronic pancreatitis and five of $10(50 \%)$ normal subjects were B72.3 positive, but MLS102 was completely negative in the latter group. Both monoclonal antibodies bound fetal pancreas diffusely. Thus, when pancreatic ductal cells have undergone malignant transformation, like the fetal pancreas, they express cell surface and secreted glycoconjugates with increased sialosyl-Tn epitopes suggesting enhanced 2-6 sialosyltransferase activity. This study shows that MLS102 is an extremely sensitive and specific tumour marker in the pancreas and that it is better than B72.3 in distinguishing pancreatic cancer from normal and chronic pancreatitis.
\end{abstract}

(Gut 1993; 34: 1722-1725)

Medical Research Centre, Nottingham City Hospital

C K Ching

R G Long

Departments of Medicine and Histopathology,

Derbyshire Royal

Infirmary, Derby

G K T Holmes

$\mathrm{S}$ W Holmes

Correspondence to:

Dr C K Ching, Medical

Research Centre, City

Hospital, Hucknall Road,

Nottingham NG5 1PB.

Accepted for publication 27 April 1993

Cellular distribution of the siaolsyl-Tn antigen identified by monoclonal antibodies MLS102 and $B 72.3$ in the pancreas

\begin{tabular}{|c|c|c|c|c|c|c|c|c|c|c|}
\hline \multirow[b]{3}{*}{ Tissue } & \multicolumn{5}{|c|}{$M L S 102$} & \multicolumn{5}{|c|}{$B 72.3$} \\
\hline & \multicolumn{3}{|c|}{ Duct } & \multicolumn{2}{|c|}{ Acini } & \multicolumn{3}{|c|}{ Duct } & \multicolumn{2}{|c|}{ Acini } \\
\hline & $S$ & IC & $M$ & $S$ & $I C$ & $S$ & $I C$ & $M$ & $S$ & $I C$ \\
\hline $\begin{array}{l}\text { Pancreatic cancer }(n=13) \\
\text { Chronic pancreatitis }(n=20) \\
\text { Normal subjects }(n=10) \\
\text { Fetal pancreas }(n=1)\end{array}$ & $\begin{array}{r}13 \\
0 \\
0 \\
1\end{array}$ & $\begin{array}{r}13 \\
0 \\
0 \\
1\end{array}$ & $\begin{array}{r}13 \\
0 \\
0 \\
1\end{array}$ & $\begin{array}{l}- \\
0 \\
0 \\
1\end{array}$ & $\begin{array}{l}- \\
0 \\
0 \\
1\end{array}$ & $\begin{array}{l}13 \\
15^{\star} \\
5 \\
1\end{array}$ & $\begin{array}{c}13 \\
15^{\star} \\
5 \\
1\end{array}$ & $\begin{array}{r}13 \\
5 \\
1 \\
1\end{array}$ & $\begin{array}{c}-\overline{15} \\
5 \\
1\end{array}$ & $\begin{array}{c}-\overline{15} \\
5 \\
1\end{array}$ \\
\hline
\end{tabular}

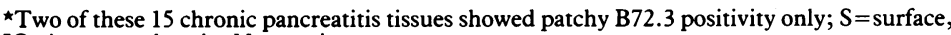
IC = intracytoplasmic, $M=$ mucin. has recently been developed against a colon cancer cell line, LS180, and has been found to bind strongly to mucin that bears a high concentration of STn epitopes." Its affinity for ovine submaxillary mucin is 130 fold higher than for rectal mucin glycoprotein, which is known to possess significantly fewer STn epitopes in its oligosaccharide side chains. In this study, we have compared and contrasted the cellular distribution of the MLS102 binding sites with B72.3 in the pancreas.

\section{Methods}

Pancreatic needle biopsy specimens were obtained from patients with pancreatic cancer $(n=13$, five well differentiated, five moderately differentiated, and three poorly differentiated adenocarcinoma); chronic pancreatitis $(n=20)$; and normal subjects $(n=10)$. Pancreas from a fetus of 15 weeks' gestation was obtained with consent for this study. These specimens were fixed in formalin and embedded in paraffin before they were sectioned for immunohistochemistry.

Sections were dewaxed, rehydrated, and then incubated in $3 \% \mathrm{H}_{2} \mathrm{O}_{2}$ to eradicate endogenous peroxidase activity. This was followed by washing the sections repeatedly $(\times 3)$ in Tris buffered saline (TBS, pH 7·6). They were then incubated in monoclonal antibody MLS102 $(5 \mu \mathrm{g} / \mathrm{ml}$, gift of Drs S Fukui and A Kurosaka, Kyoto, Japan) or B72.3 (1:160, gift of Dr J Schlom, National Cancer Institute, USA) at room temperature for one hour. Unbound antibody was removed by repeated washings $(\times 3)$ with TBS and the bound mouse monoclonal antibodies were subsequently identified by peroxidase-tagged rabbit antimouse antibody (Sigma, USA). The latter was finally visualised by incubation with 3,3 ' diaminobenzidine $/ \mathrm{H}_{2} \mathrm{O}_{2}$ containing solution. The slides were then viewed under $\times 10$ magnification and they were scored as negative if the overall staining was $<5 \%$, patchy if it was $>5 \%$ but $<30 \%$, and positive if it was $>30 \%$. Negative controls consisted of substituting normal mouse serum for MLS102 or B72.3 monoclonal antibody, which resulted in negative staining. Desialylation by using $0.5 \mathrm{mU} / \mathrm{ml}$ of Vibrio cholerae neuraminidase (Sigma, USA) at $37^{\circ} \mathrm{C}$ for 16 hours abolished both the MLS102 and B72.3 binding activity.

\section{Results (Table)}

DUCTAL TISSUES

The cell surface and the intracytoplasmic regions of all $(n=13)$ the pancreatic cancer specimens were uniformly stained by both the MLS102 

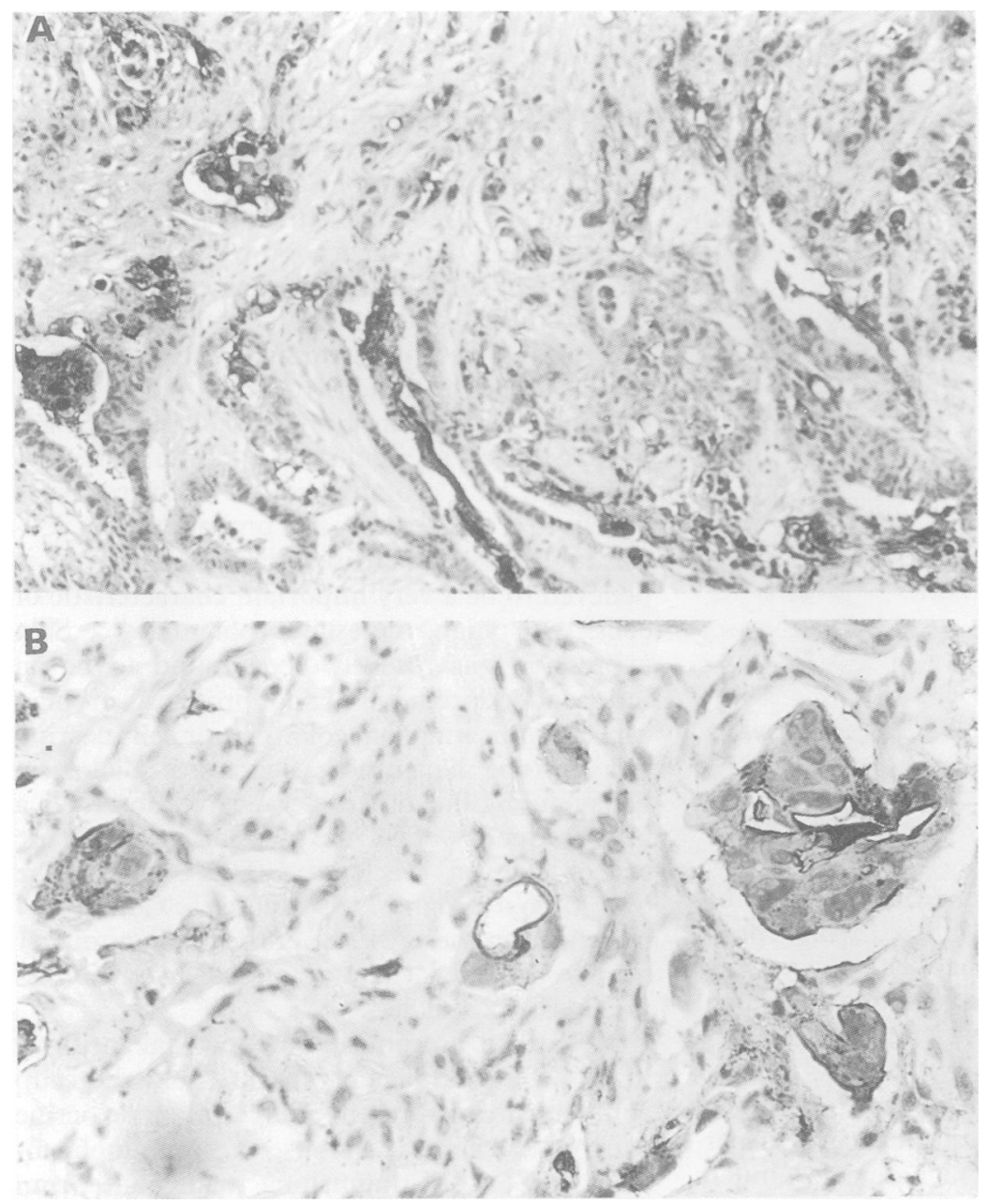

Figure 1: (A) and (B) Strong MLS102 staining (brown) is shown in the surface, cytoplasm and the intraductal mucins of these two pancreatic adenocarcinoma specimens. (Magnification: $(A) \times 16 ;(B) \times 40$.)
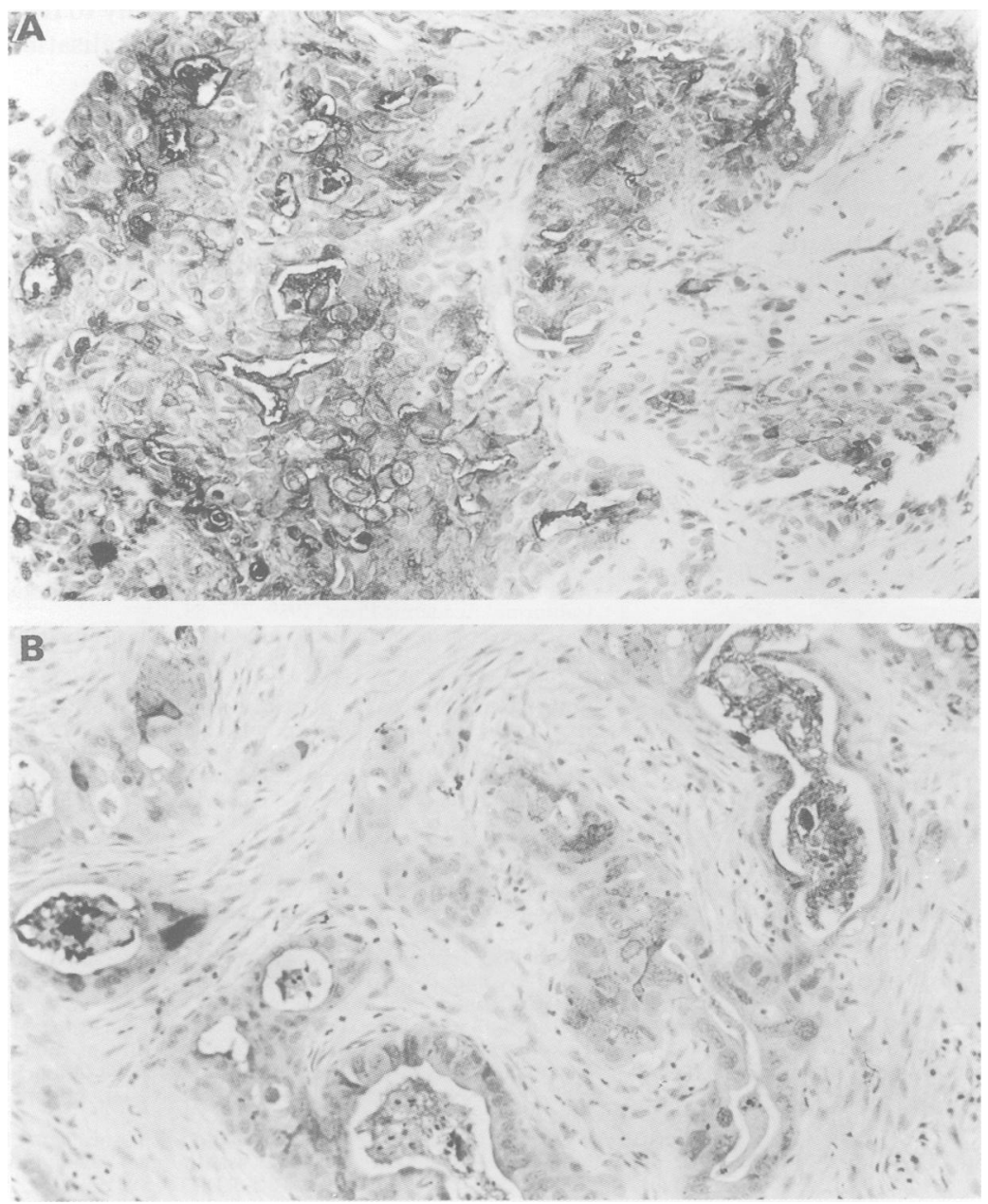

(Fig 1 (A) and (B) and B72.3 (Fig 2 (A) and (B)) monoclonal antibodies. The cellular distribution of these two STn binding monoclonal antibodies was virtually identical. Strong mucin staining (Figs 1 and 2) was similarly observed in all the 13 pancreatic cancers studied.

MLS102 was negative in the normal controls and those with benign disease but $\mathrm{B} 72.3$ bound $75 \%$ (15 of 20) of the chronic pancreatitis specimens. Thirteen of $15 \mathrm{~B} 72.3$ positive chronic pancreatitis specimens showed diffused B72.3 staining in the ductal cell surface and in the intracytoplasmic regions (Fig 3). There was also weak B72.3 positive staining in some of the intraductal mucins (Fig 3, arrowed). Half (five of 10) of the normal ductal tissues were also $B 72.3$ positive but mucins were positive in only one of these (Fig 4). The fetal pancreatic ductal tissue was positively stained by both of these monoclonal antibodies.

\section{ACINAR TISSUES}

MLS102 did not bind the normal or chronic pancreatitic acinar tissues which were positively stained by B72.3 in $50 \%$ (five of 10) of the normal control subjects and $75 \%$ (15 of 20) of the patients with chronic pancreatitis. Diffuse staining was found in the surface and the intracytoplasmic regions of five normal subjects positive for B72.3 (Fig 4). The same pattern was observed in 13 of the 15 patients with chronic pancreatitis who were positive for B72.3 (Fig 3). The remaining two showed only patchy $B 72.3$ positivity. Both the MLS102 and B72.3 monoclonal antibodies bound fetal pancreatic acinar tissue.

\section{Discussion}

MLS102 and B72.3 are both highly sensitive in detecting pancreatic cancer cells but the former is better because it is more specific. MLS102, but not B72.3, distinguishes extremely well between pancreatic cancer and normal subjects and patients with chronic pancreatitis in this study.

B72.3 has been characterised to bind STn antigen previously and there is very little difference between this antibody and TKH2 (another STn binding monoclonal antibody) in distinguishing pancreatic cancer from normal and benign controls. ${ }^{9}$ They have been shown to bind pancreatitic ductal tissues in $33 \%^{8}$ and $44 \%^{6}$ respectively in previous studies. MLS102 is a recently developed anti-STn monoclonal antibody that has been characterised to bind more strongly to antigen that bears clusters of $S T n$ epitopes. " Kurosaka et al showed that a 3.6 fold increase in the disaccharides ( $\mathrm{N}$-acetylneuraminic acid 2-6 N-acetylgalactosamine, the STn epitope) led to a 130 fold enhancement of MLS102 binding activity. B72.3 has never been shown to possess similar characteristics before. We believe this may account for the apparent difference in their specificity.

Both monoclonal antibodies bind the fetal pancreas indicating that STn antigen is a commonly present fetal antigen. The absence of MLS102 positivity in the normal adult pancreas and its reappearance after the organ has under- 


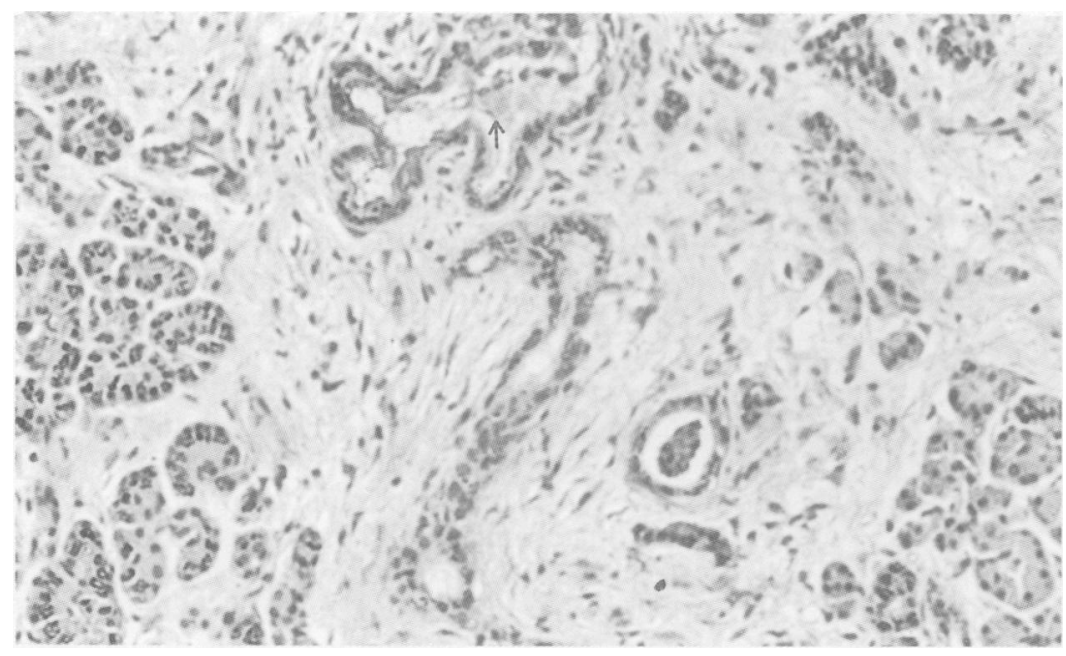

Figure 3: The pancreatic ductal and acinar tissues of this chronic pancreatitis specimen are positively stained (brown) by B72.3 monoclonal antibody. There is weak B72.3 positive mucin (arrowed) present in some of the intraductal musins. (Magnification: $\times 25$.)

gone malignant transformation suggests that there are increased STn epitopes, which probably form clusters, in the cell surface and secreted glycoconjugates in fetal and malignant pancreas suggesting the presence of enhanced 2-6 sialosyltransferase activity.

A number of blood tests have been produced to help diagnosis of pancreatic cancer. ${ }^{2}$ In particular, the CA19-9 assay which detects a mucin antigen with the sialylated Lewis ${ }^{a}$ epitope has been shown to be the best serological marker and has a sensitivity for diagnosis of pancreatic cancer of $68-93 \%$. However, false negative results are expected in patients who are Lewis ${ }^{\text {a-b- }}$, a situation which occurs in about $5 \%$ of whites. Specificity of CA19-9 radioimmunoassay is usually good, most studies reporting values of 80-93\%, but raised CA19-9 values have been reported in up to $28 \%$ of patients with acute or chronic pancreatitis or benign obstructive jaundice. This specificity is therefore too low to allow its use as a screening test. ${ }^{12}$ B72.3 assay has been shown to detect $34-74 \%$ of pancreatic cancers. ${ }^{1314}$ Sandwich enzyme linked immunosorbent assay (ELISA) is being developed by

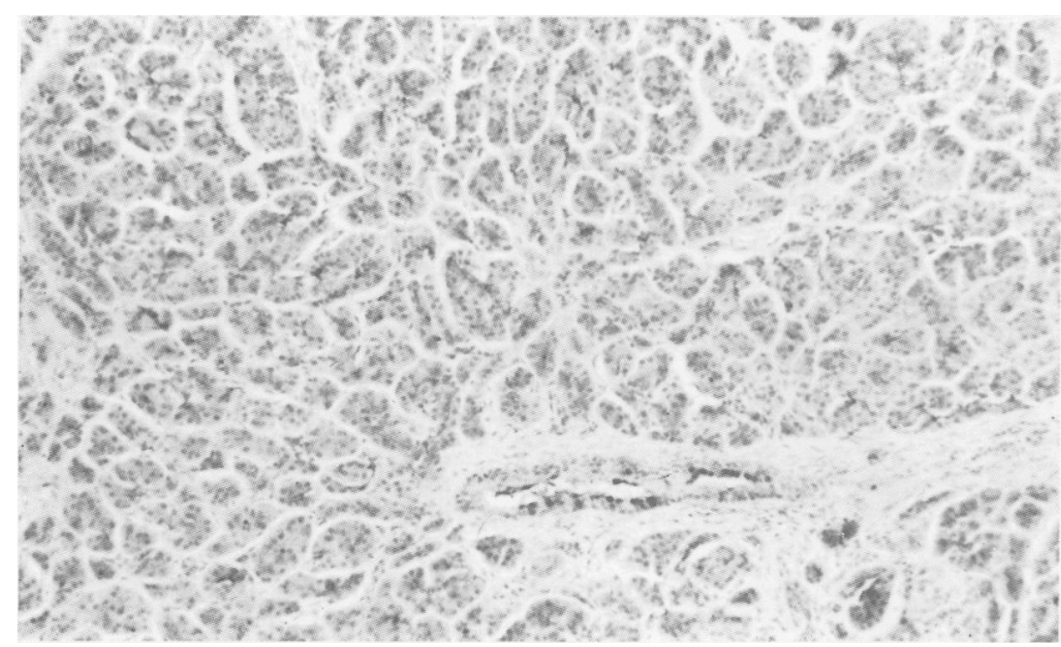

Figure 4: This normal pancreas shows strong and diffuse B72.3 positivity (brown staining) in the ductal as well as the acinar tissues. (Magnification: $\times 10$.) using the MLS102 monoclonal antibody. Its superior specificity compared with B72.3 as shown in this immunohistochemical study may suggest that a more sensitive and specific serological assay by incorporating the MLS102 monoclonal antibody may be achieved.

The study of changes in glycosylation of tissue and serum glycoconjugates in cancer have led to the discovery of tumour markers and provided valuable insights into tumour biology. Changes in cell surface or circulating glycoconjugates, or both, may have profound effects on the response of cells to growth factors and probably affect cellcell interaction. Increased sialylation of cells is believed to be a very important characteristic of cells with high metastatic potential. ${ }^{15} 16 \mathrm{STn}$ expression has recently been found to be an independent predictor of poor prognosis in colon cancer ${ }^{17}$ indicating that colon cancer cells bearing this epitope have more aggressive biological behaviour. Like others ${ }^{6-9}$ we have observed a high frequency of STn expression in pancreatic cancer cells in this study. This suggests the overall poor prognosis of pancreatic cancer may be related to the presence of the STn epitope or simply because of the increased sialylation as previously postulated. ${ }^{15} 16$

MLS102 is a promising monoclonal antibody that is directed against a STn blood group epitope which is present abundantly in exocrine pancreatic cancers. Its use in immunohistochemistry in distinguishing malignant from normal or pancreatitic controls has been outstanding when compared with a similar STn binding monoclonal antibody B72.3. Its application in serology and immunoscintigraphy to help clinical diagnosis and tumour localisation remains to be defined by further studies.

This paper was presented at the Spring 1992 British Society of Gastroenterology meeting in Sheffield. The abstract was published in Gut 1992; 33: S28.

We thank Drs S Fukui and A Kurosaka of Kyoto Sangyo University, Japan for the gift of MLS102 monoclonal antibody and Dr J Schlom of the National Cancer Institutes, USA for the B72.3 monoclonal antibody.

1 Feizi T. Demonstration by monoclonal antibodies that carbohydrate structures of glycoproteins and glycolipids are hydrate structures of glycoproteins and glycolipids

2 Rhodes JM, Ching CK. Serum diagnostic tests for pancreatic cancer. In: Neoptolemos J, ed. Baillière's clinical gastroenterology. London: Bailliere Tindall, 19??: 833-52.

3 Hakomori S. Aberrant glycosylation in tumors and tumorassociated carbohydrate antigens. In: Vande Woude GF Klein G, eds. Advances in cancer research. Vol 52. San Diego, California: Academic Press, 1989: 257-33.

4 Sell S. Cancer-associated carbohydrates identified by monoclonal antibodies. Hum Pathol 1990; 21 : 1003-19.

5 Ichihara T, Sakamoto J, Nakao A, et al. Expression of blood group-related antigens in normal and malignant pancreatic tissue correlated with genotype of the patient defined by tissue correlated with genotype of the patien
saliva glycoprotein. Cancer 1993; 71: 71-81.

6 Itzkowitz S, Kjeldsen T, Friera A, Hakomori S, Yang U, Kim YS. Expression of Tn, sialosyl-Tn and T antigens in human YS. Expression of Tn, sialosyl-Tn and T antigen

7 Schuessler MH, Pintado S, Welt S, et al. Blood group and blood-group-related antigens in normal pancreas and pancreas cancer: enhanced expression of precursor type 1 Tn and sialyl-Tn in pancreas cancer. Int $\mathcal{F}$ Cancer 1991; 47 $180-7$

8 Takiyama Y, Tempero MA, Takasaki H, et al. Reactivity of CO17-1A and B72.3 in benign and malignant pancreatic diseases. Hum Pathol 1989; 20: 832-8.

9 Kjeldsen T, Clausen J, Hirohashi S, Ogawa T, Iijima H, Hakomori S. Preparation and characterization of monoclonal antibodies directed to the tumor-associated O-linked sialosly 2-6-N-acetylgalactosaminyl (sialosyl-Tn) epitope. Cancer Res 1988; 48: 2214-20.

10 Colcher D, Horan Hand P, Nuti M, Schlom J. A spectrum of monoclonal antibodies reactive with human mammary
tumor cells. Proc Natl Acad Sci USA 1981; 78: 3199-203. 
11 Kurosaka A, Hitagawa H, Fukui S, et al. A monoclonal antibody that recognizes a cluster of a disaccharide, NeuAC 2-6 GalNAc, in mucin-type glycoproteins. $f$ Biol Chem 1988; 263: 8724-6.

12 Frebourg T, Bercoff E, Manchon N, et al. The evaluation of CA19-9 antigen level in the early detection of pancreatic cancer. A prospective study of 866 patients. Cancer 1988; 62: cancer. A prospective study of 866 patients. Cancer 1988; 62 :
$2287-90$.

13 Klug TL, Sattler MA, Colcher D, Schlom J. Monoclonal antibody immunoradiometric assay for an antigenic determinant (CA-72) on a novel panca

14 Motoo Y, Kawakami H, Watanabe H, et al. Serum sialyl-Tn antigen levels in patients with digestive cancers. Oncology 1991; 48: 321-6.

15 Yogeeswaran G, Salk PL. Metastatic potential is positively correlated with cell surface sialylation of cultured murine tumor cell lines. Science 1981; 212: 1514-6.

16 Takada A, Ohmori $\mathrm{K}$, Yoneda $\mathrm{T}$, et al. Contribution of carbohydrate antigens sialyl Lewis a and sialyl Lewis $\mathrm{x}$ to adhesion of human cancer cells to vascular endothelium. Cancer Res 1993; 53: 354-61.

17 Itzkowitz SH, Bloom EJ, Kokal WA, Modin G, Hakomori S Kim YS. Sialosyl-Tn: a novel mucin antigen associated with prognosis in colorectal cancer patients. Cancer 1990; 66: prognosis 